\title{
Risk Management Assessments of Anti-tuberculosis Adverse Drug Reaction: A Systematic Review.
}

\author{
Boubacar Traore $^{1^{*}}$, Gladys TSOUMBOU BAKANA ${ }^{1}$, Samira NANI ${ }^{1}$ et Samira HASSOUNE ${ }^{1}$ \\ 1- Epidemiology laboratory, Faculty of Medicine and Pharmacy of Casablanca, Hassan II University, Morocco. \\ 2- Health District of Kita, Mali.
}

\begin{abstract}
To improve adherence to treatment, quality of life of patients on anti-tuberculosis drugs, and prevent antibiotic resistance, we conducted this systematic review to support risk minimization actions. Methods: Medline, Scopus, and Web of Science were searched with a focus on adverse drug reactions. Two independent reviewers assessed the methodological quality of the included studies using criteria defined by the Newcastle Ottawa Scale. Results: Seven studies were included, and four risk management strategies were identified (psychological intervention, drug dose reduction with or without prescription of adjunctive drugs, drug switching, permanent or temporary drug discontinuation). The strategies adopted were dependent on the nature and severity of the adverse events. All drugs responsible for serious adverse effects were changed or discontinued. Conclusions: The results highlight the relatively low frequency of adverse events leading to permanent discontinuation of 1st-line anti-tuberculosis drugs, but also emphasize the high incidence of adverse events leading to permanent discontinuation of cycloserine.
\end{abstract}

\section{Introduction}

Antibiotic resistance, and specifically TB drug resistance, has become a global threat with potential consequences for human health, animal health, and the environment. Indeed, 206,030 cases of multidrugresistant tuberculosis (a 10\% increase from 2018) have been identified among the 10 million cases of tuberculosis detected and reported in 2019 . In addition, 1.4 million TB deaths were reported in 2019 [1].

Antibiotic resistance results from poor adherence to treatment, insufficient dosage, pharmacokinetic interaction or insufficient antibacterial activity leading to inappropriate use and selection of resistant mutants [2].

If dose reduction (insufficient dosage) is an attitude adopted by clinicians to manage adverse effects, patients adopt poor compliance to manage adverse effects [3].

Indeed, the treatment of tuberculosis and multidrugresistant tuberculosis requires long-term therapy with a combination of several drugs. Adverse effects are much more common with these drugs, especially those used for the treatment of MDR-TB.

The adverse effects can affect the quality of care, but also the physical, organic integrity of patients and psycho-affective and social, directly interfering with the quality of life [4]. They can lead to major morbidity, including blindness, deafness, myelosuppression, renal failure or liver failure, resulting in hospitalization or even death, as well as treatment interruption or failure $[3,5,6]$. Most of these adverse events are minor and can be treated without interruption of therapy.

The timing, disease course, and semiology of the adverse events can help determine the drugrelatedness of these suspected adverse events [3].

In addition to causality due to the mechanisms of action of anti-tuberculosis drugs, various factors are associated with the occurrence of adverse effect, including dose and time of day the drug is administered, patient age, nutritional status, presence of preexisting diseases or dysfunctions such as impaired liver function, impaired renal function, human immunodeficiency virus (HIV) co-infection, and alcoholism [3].

A better understanding of the toxicity of all drugs used to treat tuberculosis, a good bacteriological diagnosis, a better management of adverse effects associated with therapeutic compliance is essential to prevent resistance to anti-tuberculosis drugs; improve the management and quality of life of patients.

To support risk minimization actions, we conducted this systematic review to identify and evaluate different strategies for managing the adverse effects of anti-tuberculosis drugs.

\section{Method:}

\subsection{Information sources and search strategies}

The different steps of our literature search methodology were based on the recommendations of the PRISMA 2020 grid ("Preferred Reporting Items for Systematic Reviews and MetaAnalyses") [7] and a didactic article [8].

*corresponding author : $\underline{\text { drbtraore@gmail.com }}$ 
The literature search was initially performed on 05 May 2019 and updated on 04 August 2020 in PubMed (including MEDLINE), Scopus, and Web of Sciences using the term ("Tuberculosis/therapy") AND "DrugRelated Side Effects and Adverse Reactions"). The term was adapted to the thesaurus of each database and no date restrictions were made. However, the search was limited to adults over 19 years of age and to articles in French or English.

\subsection{Eligibility criteria}

The management of adverse events in the treatment of extrapulmonary tuberculosis, latent tuberculosis, co-infections (HIV, Hepatitis) and association with other diseases such as diabetes; was excluded from our research. Indeed, the treatment of pulmonary tuberculosis has an adverse event profile that can be very different (in terms of severity and frequency) from the treatment of extra-pulmonary tuberculosis, latent tuberculosis, and co-infections.

The management of adverse effect of pulmonary TB treatment in children has also been excluded. Children are a vulnerable population with a very different immune system than adults. This vulnerability is increased with tuberculosis, and the immune response of these children to antituberculosis treatments can be fatal and the side effect profile different from that of adults.

Due to incomplete results, conference proceedings and abstracts were not included in the search; nor were: opinion articles, editorials, guidelines, case reports, and studies not reporting on TB treatment (studies addressing laboratory tests, laboratory research, pharmacokinetic studies, etc.).

Although systematic reviews and meta-analyses identified in the screening were not included, they were used to search for additional references to include in the search.

\subsection{Study selection process}

The selection was made by two independent reviewers (TRAORE $B$ and TSOUMBOUBAKANA G) using a standardized selection grid. A third reviewer (HASSOUNE S) was called upon in case of disagreement.

The following terms: adverse or toxicity or side effects or safety or tolerability AND drug-resistant or multi-drug resistant or first-line treatment or secondline treatment or antituberculosis drug AND tuberculosis or TB; were searched in the titles and/or abstracts. The article was excluded from further review if it was clear from the title or abstract that the article did not meet the inclusion criteria. After this step, the full text of articles whose abstracts were not detailed enough to determine eligibility, as well as the full text of included articles, were searched and examined to see if they described the strategy for managing the adverse effects of anti-tuberculosis drugs according to the severity of the adverse effects.
Only those articles that reported on adverse event management strategies by adverse event profile and severity were included in the qualitative synthesis.

The article with the most information on adverse event management was included when the same patients were reported in multiple eligible manuscripts.

\subsection{Data Extraction}

Adverse drug reactions were extracted and classified according to the SOC (System Organ Class) of the MedDRA (Medical Dictionary for Regulatory Activities) classification [9] and the severity of the adverse reactions. While the SOC classification was based on the High-Level Terms (HLT) identified in the article, the severity of the adverse events was extracted from the authors' estimates. However, when severity was not mentioned, the adverse event was considered serious if it resulted in death, hospitalization or prolongation of hospitalization, persistent significant disability, or congenital anomaly; or if it was life-threatening to the patient [10].

Available data on suspected drugs and adverse event management strategies were also extracted.

\subsection{Risk of bias assessment}

The methodological quality of the included studies was assessed using the criteria defined by the Newcastle Ottawa Scale [11] by two independent reviewers (TSOUMBOU-BAKANA G, TRAORE B).

\subsection{Data Analysis}

Extracted data were entered into Excel 2016 spreadsheet and then exported into RStudio software (version 1.2.5033) for analysis.

\section{Results:}

\subsection{Study selection}

Our search strategies on PubMed, Scopus, and Web of science identified 439 references, of which 27 were found to be duplicates. A total of 32 potentially relevant articles on the risk management of adverse drug reactions to anti-tuberculosis drugs were identified after an initial screening based on the titles and abstracts of candidate articles. After the exclusion of 4 articles for not having access to the full text and the second screening, 28 articles were examined on the eligibility criteria after which 21 articles were excluded. Figure 1 summarizes the article selection processes.

Only the remaining 7 articles [12-18] were included in the systematic review.

\subsection{Characteristics of the included studies}


Of the 7 included articles 5 were retrospective crosssectional studies $[12,13,16-18]$ and 2 were prospective cohorts [14,15].

These studies were conducted between 2006 and 2017 in India [12,14,17], Vietnam [13], Pakistan [15], Nepal [16] and South Korea [18]; and published in English.

They involved 1159 patients with pulmonary tuberculosis, including 554 men and 279 women. The sex of the participants was not defined in one study [16].

This population consisted of patients with treatmentsensitive tuberculosis $[16,17]$ and multidrug-resistant tuberculosis $[12-15,18]$.

The mean age of participants ranged from $29.3 \pm 9.3$ years to $44.3 \pm 12.88$ years, and there was no information on the mean age of participants in one study [17].

The main objectives of these studies were to investigate the occurrence of adverse events under anti-tuberculosis treatment [12-18], and risk factors for adverse effects of anti-tuberculosis drugs [14,15].

\subsection{Assessment of risk of bias}

The studies were heterogeneous with scores ranging from 2 to 7 on the NOS scale. Two studies had a medium risk of bias and the others had a high risk of bias (Table 1).

\subsection{Summary of results}

In the overall population of 1159 patients, 938 adverse events under anti-tuberculosis treatment were reported, of which $93.3 \%(875 / 938)$ under treatment of multidrug-resistant tuberculosis and 6.7\% (63/938) under treatment of susceptible tuberculosis.

The time to onset of adverse effects was reported only in two articles $[12,18]$ and ranged on average from 25 days to $6.8 \pm 4.1$ months.

All studies reported gastrointestinal disorders (nausea, vomiting, abdominal pain, gastritis, diarrhea), central nervous system disorders (peripheral neuropathy, hearing impairment, headache, dizziness...), hepatotoxicity, nephrotoxicity, arthralgia, and skin reactions with most disorders being minor to moderate.

Psychiatric disorders [12-14,18], metabolic and nutritional disorders $[14,15,18]$, and ocular neuritis $[14-16,18]$ have also been reported by the authors.

The most frequently incriminated drugs were: ethionamide (Eto) [14,16] (gastrointestinal disorders, ocular neuritis, and metabolic and nutritional disorders, hepatotoxicity); para-aminosalicylic acid (PAS) $[15,18]$ (gastrointestinal disorders, metabolic disorders, and hepatotoxicity); kanamycin [15] (central nervous system and hearing disorders); levofloxacin [15] (gastrointestinal disorders, central nervous system and hearing disorders, skin reactions); pyrazinamide [15] (hepatotoxicity, arthralgias) and cycloserine [13-16,18] (central nervous system and hearing disorders, psychiatric disorders, nephrotoxicity).

The management of minor adverse drug reactions did not require interruption of treatment. It did, however, require psychological support and, in rare cases, the prescription of auxiliary drugs $[14,16,18]$.

Moderate adverse effects of anti-tuberculosis drugs were mainly managed by reducing the dose of the suspect drug with or without prescriptions for adjuvant drugs.

Simple and moderate gastrointestinal disorders did not require either discontinuation of treatment or reduction of the dose of drugs. They sometimes required the prescription of auxiliary drugs.

A permanent discontinuation of the suspect drug, and a change of treatment protocol (change of the suspect drug and/or all other drugs to other anti-tuberculosis drugs) was carried out in the case of serious adverse events).

Psychiatric disorders, metabolic disorders, central nervous system disorders, ocular neuritis, ototoxicities have most often required permanent discontinuation of the suspect drug, and a change of therapeutic protocol. 


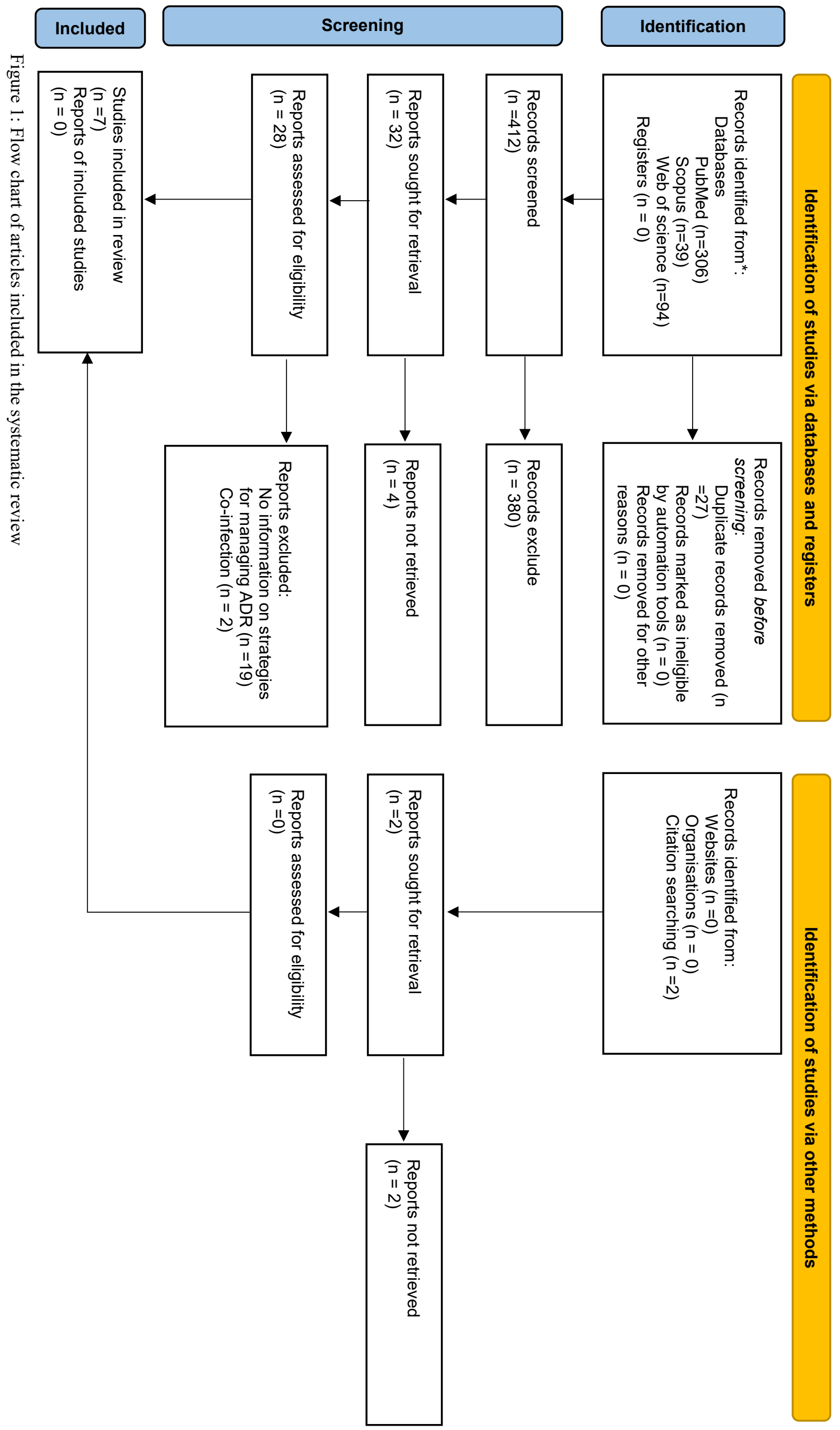




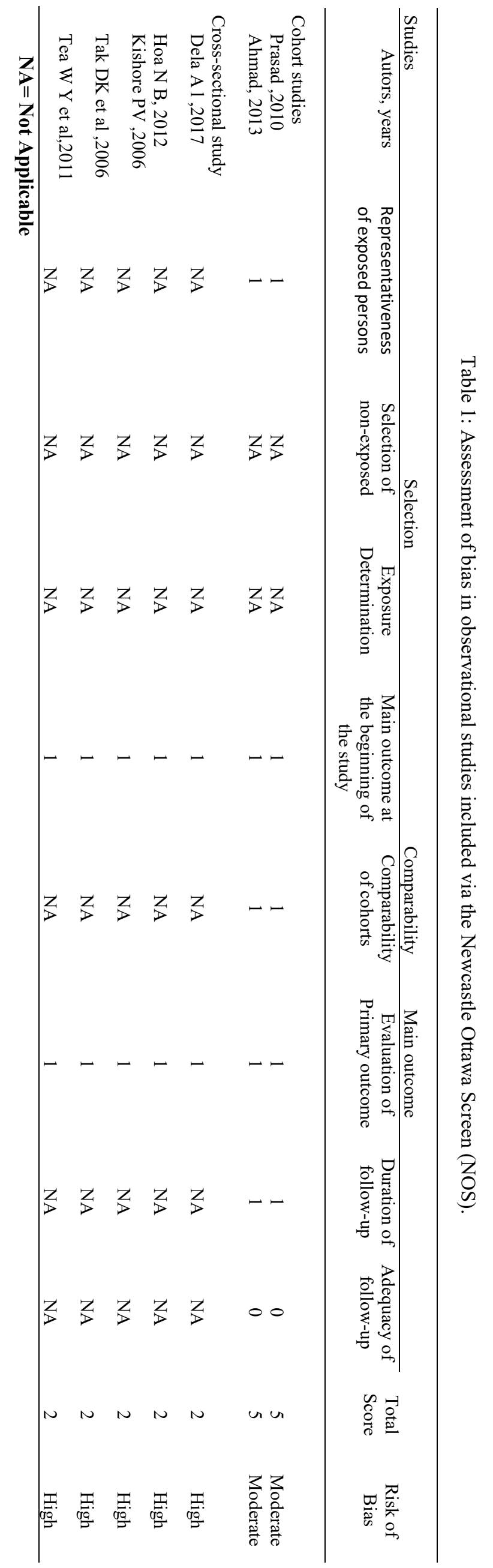




\section{Discussion}

Although tuberculosis is a public health problem, studies on the adverse effects of anti-tuberculosis drugs are rare, and few are conducted in Africa. In addition, imputability analysis is generally not performed, which may underestimate the magnitude of adverse drug reactions. Indeed, of the seven articles included, only three performed imputability analysis $[15,16,17]$.

Most patients lived in India $[13,15,18]$, Vietnam [14], Pakistan [16], Nepal [17] and South Korea [18]. Indeed, India and Pakistan are among the nine countries (India, China, Indonesia, Philippines, Pakistan, Nigeria, Bangladesh, and South Africa) most affected by TB in 2019 [1].

\subsection{Adverse effects of anti-tuberculosis drugs:}

The adverse effects of anti-tuberculosis drugs were generally observed under 2 nd line treatment. This could be explained by the combination of more toxic molecules for the treatment of MDR-TB $[19,20]$. It is therefore necessary to train health workers to recognize and systematically investigate the adverse effects of anti-tuberculosis drugs to ensure successful treatment and improve the quality of life of patients undergoing treatment.

Serious adverse effects were generally rare and more frequent in second-line treatment compared to firstline treatment. Törün in 2005 [21] made the same observation.

As noted by other authors [22,23], hepatotoxicity was the most frequently reported serious adverse event under first-line antituberculosis chemotherapy.

\subsection{Management of adverse drug reactions}

\subsubsection{Minor adverse effect:}

Apart from psychological management to reassure patients, minor adverse events did not require a reduction in dosage, discontinuation of treatment, or a change in the suspected drug. In fact, psychological management of adverse events is strongly recommended by the World Health Organization [49] in the guidelines for TB programs in the management of drug-resistant tuberculosis.

\subsubsection{Moderate adverse effects.}

While the management of moderate side effects of first-line anti-tuberculosis chemotherapy has consisted of temporary discontinuation followed by reintroduction of the suspect drug, the management of second-line chemotherapy has consisted of dose reduction with or without prescription of adjuvant therapies.

The adverse effects of most second-line antituberculosis drugs are dose-dependent [24] and they can therefore be managed by reducing the dose of the suspect drug while respecting the therapeutic efficacy window to avoid therapeutic failures and the appearance of antibiotic resistance [25].

\subsubsection{Serious adverse effects:}

The International Union Against Tuberculosis and Lung Disease guidelines recommend dose reduction or discontinuation and replacement of the suspect drug when the adverse reaction is life-threatening or likely to cause permanent injury. These adverse events require an experienced clinician to identify the suspected drug, reduce its dose or discontinue it and replace it with an equivalent drug if discontinuation is definitive. Discontinuation and replacement of the suspect drug should be considered a last resort after attempting to manage the adverse reaction in question with adjunctive medications [26].

Cycloserine was discontinued and replaced in all studies. Indeed, several authors have reported the relative psychiatric toxicity of cycloserine [27-28].

The strategy for the management of serious adverse events adopted in the seven articles included in this review of the literature was consistent with these principles.

\subsection{Limitations}

This review of the literature is limited to adult patients with active pulmonary tuberculosis who are not co-infected with HIV. Because of the particularities of their management, children and HIV-infected subjects were excluded from our review. Our search was also limited to studies reporting management strategies for adverse drug reactions according to severity. It was also limited to the frequencies of adverse events reported on antituberculosis drugs; it was difficult to study the occurrence of adverse events per patient and the accountability of individual adverse events. This information was not available in many of the included studies.

\section{Conclusions}

The results of this systematic review suggest that close monitoring of adverse events is important for patients treated for multidrug-resistant tuberculosis, and also highlight the urgent need for safer and better-tolerated drugs to improve patient management and quality of life, as well as treatment outcomes; and to prevent TB drug resistance. Indeed, they highlight the relatively low incidence of adverse events leading to permanent discontinuation of anti-tuberculosis drugs, but also emphasize the high incidence of adverse events leading to permanent discontinuation of cycloserine.

\section{References}

[1]. Organisation Mondiale de la Santé. Rapport sur la tuberculose dans le monde 2020: résumé d'orientation. (Organisation mondiale de la Santé, 2020). 
[2]. Davies, J. \& Davies, D. Origins and Evolution of Antibiotic Resistance. Microbiol. Mol. Biol. Rev. MMBR 74, 417-433 (2010).

[3]. Edwards, I. R. \& Aronson, J. K. Adverse drug reactions: definitions, diagnosis, and management. The Lancet 356, 1255-1259 (2000).

[4]. Valadares, R. M. C., Carvalho, W. da S. \& Miranda, S. S. de. Association of adverse drug reaction to anti-tuberculosis medication with quality of life in patients in a tertiary referral hospital. Rev. Soc. Bras. Med. Trop. 53, e20190207 (2019).

[5]. Forget, E. J. \& Menzies, D. Adverse reactions to first-line antituberculosis drugs. Expert Opin. Drug Saf. 5, 231-249 (2006).

[6]. Tan, W. C., Ong, C. K., Kang, S. C. L. \& Razak, M. A. Two years review of cutaneous adverse drug reaction from first line anti-tuberculous drugs. Med. J. Malaysia 62, 143-146 (2007).

[7]. Page, M. J. et al. The PRISMA 2020 statement: an updated guideline for reporting systematic reviews. BMJ 372, n71 (2021).

[8]. Zoukal, S., Bakana, G. T., Nani, S. \& Hassoune, S. Démarche d'élaboration d'une revue systématique. Rev. Marocaine Santé Publique 6, (2019)

[9]. MedDRA | Medical Dictionary for Regulatory Activities. https://www.meddra.org/.

[10]. Bégaud, B. Dictionnaire de pharmacoépidémiologie. (1995).

[11]. Hartling, L. et al. Decision Rules for Application of the Newcastle-Ottawa Scale. Validity and Inter-Rater Reliability Testing of Quality Assessment Instruments [Internet] (Agency for Healthcare Research and Quality (US), 2012).

[12]. Arif I Dela, Tank, N. K. D., Singh, A. P. \& Piparva, K. G. Adverse drug reactions and treatment outcome analysis of DOTS-plus therapy of MDRTB patients at district tuberculosis centre: A four year retrospective study. Lung India Off. Organ Indian Chest Soc. 34, 522-526 (2017).

[13]. Hoa, N. B., Nhung, N. V., Khanh, P. H., Hai, N. V. \& Quyen, B. T. T. Adverse events in the treatment of MDR-TB patients within and outside the NTP in Pham Ngoc Thach hospital, Ho Chi Minh City, Vietnam. BMC Res. Notes 8, 809 (2015).

[14]. Prasad, R. et al. Frequency of adverse events observed with second-line drugs among patients treated for multidrug-resistant tuberculosis. Indian J. Tuberc. 63, 106-114 (2016).

[15]. Ahmad, N. et al. Occurrence, Management, and Risk Factors for Adverse Drug Reactions in Multidrug Resistant Tuberculosis Patients. Am. J. Ther. 25, e533-e540 (2018).

[16]. P V, K., Palaian, S., Ojha, P. \& P R, S. Pattern of adverse drug reactions experienced by tuberculosis patients in a tertiary care teaching hospital in Western Nepal. Pak. J. Pharm. Sci. 21, 51-56 (2008).

[17]. Tak, D. K., Acharya, L. D., Gowrinath, K., Rao Padma, G. M. \& Subish, P. Safety evaluation of antitubercular therapy under Revised National Tuberculosis Control Programme in India. J. Clin. Diagn. Res. 3, 1395-1401 (2009).

[18]. Yang, T. W. et al. Side effects associated with the treatment of multidrug-resistant tuberculosis at a tuberculosis referral hospital in South Korea: A retrospective study. Medicine (Baltimore) 96, e7482 (2017).

[19]. Ormerod, L. P. \& Horsfield, N. Frequency and type of reactions to antituberculosis drugs: Observations in routine treatment. Tuber. Lung Dis. 77, 37-42 (1996).

[20]. Aouam, K. et al. Les effets indésirables des antituberculeux: épidémiologie, mécanismes et conduite à tenir. Médecine Mal. Infect. 37, 253-261 (2007)

[21]. Törün, T. et al. Side effects associated with the treatment of multidrug-resistant tuberculosis. Int. J. Tuberc. Lung Dis. 9, 1373-1377 (2005).

[22]. Kouider, M. E. R. I. E. O., Benkhaled, I. D. R. I. S. \& Kheloui, Y. A. C. I. N. Les effets secondaires majeurs aux antituberculeux. Rev. Fr. Allergol. 59, 283-284 (2019).

[23]. Yee, D. et al. Incidence of Serious Side Effects from First-Line Antituberculosis Drugs among Patients Treated for Active Tuberculosis. Am. J. Respir. Crit. Care Med. 167, 1472-1477 (2003).

[24]. World Health Organization. Companion Handbook to the WHO Guidelines for the Programmatic Management of Drug-resistant Tuberculosis.

Http://apps.who.int/iris/

bitstream/10665/75146/1/9789241548441_eng.pdf.

[25]. Organisation Mondiale de la Santé. Le traitement de la tuberculose : principes à l'intention des programmes nationaux. WHO/TB/97.220,

[26]. Union internationale contre la tuberculose et les maladies respiratoires. Guide PIH à la gestion médicale de la tuberculose multirésistante. https://www.pih.org/practitioner-resource/pihguide-to-the-medical-management-of-multidrugresistant-tuberculosis.

[27]. Intini, E., Kishore, G., Richeldi, L. \& Udwadia, Z. F. Neuropsychiatric reactions induced by cycloserine in the treatment of multidrug-resistant tuberculosis: what an Indian female patient tells us. BMJ Case Rep. 12, (2019).

[28]. Court, R. et al. Neuropsychiatric toxicity and cycloserine concentrations during treatment for multidrug-resistant tuberculosis. Int. J. Infect. Dis. IJID Off. Publ. Int. Soc. Infect. Dis. 105, 688-694 (2021) 